\title{
Mutations in the potassium channel subunit KCNE1 are associated with early-onset familial atrial fibrillation
}

\author{
Morten S Olesen ${ }^{1,2 \dagger}$, Bo H Bentzen ${ }^{1,3 \dagger}$, Jonas B Nielsen ${ }^{1,2}$, Annette B Steffensen 1,3, Jens-Peter David ${ }^{1,3}$, \\ Javad Jabbari ${ }^{1,2}$, Henrik K Jensen ${ }^{4}$, Stig Hauns $\varnothing^{1,2,5}$, Jesper H Svendsen ${ }^{1,2,5}$ and Nicole Schmitt ${ }^{1,3^{*}}$
}

\begin{abstract}
Background: Atrial fibrillation (AF) is the most common arrhythmia. The potassium current $I_{\mathrm{Ks}}$ is essential for cardiac repolarization. Gain-of-function mutations in $K_{V} 7.1$, the pore-forming $\alpha$-subunit of the $I_{K_{s}}$ channel, have been associated with AF. We hypothesized that early-onset lone AF is associated with mutations in the $I_{\mathrm{Ks}}$ channel regulatory subunit KCNE1.

Methods: In 209 unrelated early-onset lone AF patients ( $<40$ years) the entire coding sequence of KCNE1 was bidirectionally sequenced. We analyzed the identified KCNE1 mutants electrophysiologically in heterologous expression systems.

Results: Two non-synonymous mutations G25V and G60D were found in KCNE1 that were not present in the control group ( $n=432$ alleles) and that have not previously been reported in any publicly available databases or in the exom variant server holding exom data from more than 10.000 alleles. Proband 1 (female, age 45, G25V) had onset of paroxysmal AF at the age of 39 years. Proband 2 (G60D) was diagnosed with lone AF at the age of 33 years. The patient has inherited the mutation from his mother, who also has AF. Both probands had no mutations in genes previously associated with AF. In heterologous expression systems, both mutants showed significant gainof-function for $I_{\mathrm{ks}}$ both with respect to steady-state current levels, kinetic parameters, and heart rate-dependent modulation.

Conclusions: Mutations in $K_{V} 7.1$ leading to gain-of-function of $I_{K s}$ current have previously been described in lone $A F$, yet this is the first time a mutation in the beta-subunit KCNE1 is associated with the disease. This finding further supports the hypothesis that increased potassium current enhances AF susceptibility.
\end{abstract}

Keywords: Lone AF, Genetics, KV7.1, KCNE1, I Ks current

\section{Background}

Atrial fibrillation (AF) is the most prevalent sustained cardiac arrhythmia. It is responsible for considerable morbidity and mortality, and its population prevalence has reached epidemic proportions, affecting almost seven million patients in the European Union and the USA combined [1-4].

\footnotetext{
* Correspondence: nschmitt@sund.ku.dk

+ Contributed equally

'The Danish National Research Foundation Centre for Cardiac Arrhythmia, Copenhagen, Denmark

Full list of author information is available at the end of the article
}

In most cases $\mathrm{AF}$ is associated with cardiac risk factors such as hypertensive, ischemic, and/or structural heart disease $[1,5]$. However, $10-20 \%$ of patients suffering from AF are younger than 60 years of age and lack the traditional risk factors for AF. These patients are considered as having "lone" AF [2]. The mechanisms underlying $\mathrm{AF}$ are not fully understood, but a heterogeneous model based on the interaction of multiple substrates and triggers is thought to underlie the pathophysiology of the disease. However, early-onset lone AF has been suggested to be a primary electrical disease caused by disturbances in ionic currents. Of note, a genetic cause
Ciomed Central

() 2012 Olesen et al; licensee BioMed Central Ltd. This is an Open Access article distributed under the terms of the Creative Commons Attribution License (http://creativecommons.org/licenses/by/2.0), which permits unrestricted use, distribution, and reproduction in any medium, provided the original work is properly cited. 
of these types of electrical disturbances is becoming increasingly recognized [6].

Identification of the genetic components of AF, and the importance of single nucleotide polymorphisms (SNPs) was recently shown in genome-wide association studies indicating that common variants also play a role in the development of AF $[7,8]$. This association between SNPs and AF was strongest in patients diagnosed at a younger age. There is evidence that variations in genes encoding ion channel subunits are associated with familial predisposition for AF. Several genetic reports have revealed mutations associated with AF in cardiac ion channels and accessory subunits [6]. Most of these studies show that gain- or loss-of-function mutations in the genes encoding proteins contributing to cardiac depolarization, e.g. $S C N 1-3 B$ (involved in $\mathrm{I}_{\mathrm{Na}}$ ) $[6,9]$, or cardiac repolarisation, e.g. KCNQ1 $\left(\mathrm{I}_{\mathrm{Ks}}\right), K C N H 2$ $\left(\mathrm{I}_{\mathrm{Kr}}\right), K C N J 2\left(\mathrm{I}_{\mathrm{K} 1}\right)$ can lead to increased susceptibility to AF [6]. These results support the two current conceptual models for AF. The first one being that cardiac action potential shortening functions as a substrate for re-entry wavelets in the atria $[10,11]$ the second one proposing that a prolonged effective refractory period enhances the propensity for early after depolarization, and thereby increasing the susceptibility to AF [12].

$\mathrm{K}_{\mathrm{V}} 7.1$, the $\alpha$-subunit of the $\mathrm{I}_{\mathrm{Ks}}$ current, has repeatedly been associated with AF [6]. Co-expression of its regulatory $\beta$-subunit KCNE1 changes the biophysical properties of the $K_{V} 7.1$ channel dramatically [13]. We hypothesized that early-onset lone AF is associated with mutations in KCNE1.

\section{Methods}

An expanded Methods section is available in Additional file 1.

\section{Study subjects}

Consecutive patients with lone AF and onset of AF before 40 years (i.e. absence of clinical or echocardiographic findings of other cardiovascular diseases, hypertension, metabolic or pulmonary diseases) were included from eight hospitals in the Copenhagen region of Denmark [9]. Healthy controls (216) were recruited from blood donors. The study conforms to the principles outlined in the Declaration of Helsinki and was approved by the Scientific Ethics Committee of Copenhagen and Frederiksberg (Protocol reference number KF 01313322). All included patients gave written informed consent.

\section{Mutation screening}

Genomic DNA was extracted from blood samples using the QIAamp DNA Blood Mini Kit (QIAGEN, Hilden, Germany). Oligonucleotide primers for exons and splice junctions were designed using the known sequence of human KCNE1 [Genbank:NG_009091.1]. All primers were designed with M13 tail sequences. DNA fragments amplified by Touchdown PCR were analyzed using a high-resolution melting curve analysis (Light Scanner, Idaho Technology, UT, USA). Fragments with melting curves differing from the curves of wild-type DNA were purified and directly sequenced using M13 primers and Big Dye chemistry (DNA analyzer 3730, Applied Biosystems, CA, USA). The identified variants were validated by the resequencing of a second PCR product.

A group of 216 ethnically matched healthy controls was screened employing high resolution melting curve analysis (Light Scanner, Idaho technology, Salt Lake City, USA), and bidirectional sequencing of genes previously associated with AF was performed (Additional file 1). A mutation was considered suspected being disease causing if criteria previously defined were met [9].

\section{Molecular biology}

Site-directed mutagenesis introducing the mutations G25V (c.74 C > T) and G60D (c.179 G > A) into human KCNE1 cDNA [Genbank:NM_000219.3] and in vitro transcription were performed using standard procedures. For a detailed description please refer to the Additional file 1.

\section{Heterologous expression studies}

We employed two-electrode voltage-clamp experiments using Xenopus laevis oocytes expressing wild-type or mutant $\mathrm{I}_{\mathrm{Ks}}$ and patch-clamp experiments using mammalian cells. A detailed description is available in Additional file 1 .

\section{Data analysis}

Data analysis was performed with Igor Pro (Wavemetrics, Lake Oswego, OR, USA) and GraphPad Prism (GraphPad Software Inc., San Diego, CA, USA). I/Vcurves were constructed by measuring the current at the end of a voltage-step to potentials ranging from -100 to $+60 \mathrm{mV}$ for TEVC recordings or $+40 \mathrm{mV}$ for patchclamp experiments, respectively. The data was plotted against the corresponding membrane potentials. Similarly, peak tail-currents, measured at $-120 \mathrm{mV}$ for TEVC or $-40 \mathrm{mV}$ for patch-clamping experiments following the depolarizing step, were plotted against the membrane potential of the depolarizing step to construct the activation curves. A Boltzmann function $\left(I / I_{\max }=I_{\min }+\right.$ $\left.\left(I_{\text {max }}-I_{\text {min }}\right) /\left(1+\exp \left(\left(\mathrm{V}_{50}-\mathrm{V}\right) / \mathrm{k}\right)\right)\right)$ was fitted to the activation curves to obtain the potential of half-maximal activation $\left(\mathrm{V}_{50}\right)$ and the slope factor $(\mathrm{k})$. Activation of $\mathrm{K}_{\mathrm{V}} 7 \cdot 1 / \mathrm{KCNE} 1$ channels results in sigmoidal activation current traces. In order to compare the activation kinetics we determined the time needed to reach the 
half-maximal current level $\left(t_{1 / 2}\right)$. An estimate of the values of the time constants of channel deactivation $(\tau)$ was obtained by fitting a mono-exponential function to tail-current traces measured at -140 to $-40 \mathrm{mV}$ following a voltage-step to $+40 \mathrm{mV}$ for TEVC or $0 \mathrm{mV}$ for patchclamp experiments.

The frequency dependence of conduction was investigated using three different voltage protocols $(60,120$, and $180 \mathrm{bpm}$ ). The amount of charge conducted by the $\mathrm{I}_{\mathrm{Ks}}$ channel complex was calculated by integrating the area under the curve in the first $130 \mathrm{~ms}$ after the capacitive spike $(10 \mathrm{~ms})$ of the pulse at the 7 th second. At this time point the current amplitude had reached a steadystate for all pacing frequencies tested. The amount of charge carried was normalized to the charge carried at $60 \mathrm{bpm}$.

Data are represented as mean \pm SEM, unless otherwise indicated. Unpaired t-tests or ANOVA followed by Tukey's method of multiple comparisons were used as appropriate to compare the wild-type and mutated $\mathrm{I}_{\mathrm{Ks}}$ channel complex. P-values below 0.05 were considered statistically significant.

A detailed description of all methods used is available in Additional file 1.

\section{Results}

\section{Study cohort}

The study population consisted of 209 patients with onset of AF ranging from 16 to 39 years, without any concomitant disease. A control population of 216 healthy blood donors was collected (52\% male gender, median age of 39 years (interquartile range 30-48 years)). Healthy controls were included after clinical evaluation and ECG recording. All included individuals were of Danish/Caucasian ethnicity. Clinical data of the study population is shown in Table 1[14].

\section{Mutation screening}

Direct DNA sequencing of $K C N E 1$ from the 209 index patients revealed two non-synonymous mutations in KCNE1 (c.74 g > t, G25V; c.179 G > A, G60D) (Figure 1). The mutations were not present in the control group $(\mathrm{n}=216)$, and have not previously been reported to be associated with AF. All genetically affected probands were heterozygous carriers. The positions G25 and G60 in $K C N E 1$ are highly conserved across species suggesting a functional importance (Additional file 1: Figure S1).

\section{Clinical data}

Proband 1 (female, age 45) was diagnosed with paroxysmal AF at the age of 39 years. The sinus rhythm ECG showed an incomplete right bundle branch block (IRBBB; defined as an extra r'-wave in lead V1 and/or
Table 1 Clinical characteristics of the lone AF population ( $\mathrm{n}=209$ )

\begin{tabular}{ll}
\hline Median age of onset, y (IQR) & $31.5(26-36)$ \\
Male gender, \% & 82 \\
Height, $\mathrm{cm}$ & $183 \pm 9$ \\
Weight, $\mathrm{kg}$ & $89 \pm 17$ \\
BMl, $\mathrm{kg} / \mathrm{m}^{2}$ & $26.7 \pm 4.6$ \\
Blood Pressure, $\mathrm{mmHg}$ & \\
$\quad$ Systolic & $131 \pm 13$ \\
$\quad$ Diastolic & $78 \pm 9$ \\
AF type & \\
$\quad$ Paroxysmal, \% & 55.9 \\
$\quad$ Persistent, \% & 35.9 \\
$\quad$ Permanent, \% & 8.2 \\
Family history of AF & \\
$\quad 1^{\text {st }}$ degree relatives with AF, \% & 31 \\
\hline
\end{tabular}

All numbers are reported as mean \pm standard-deviation unless otherwise noted. $I Q R$ Interquartile range.

V2 [15]) but was otherwise normal (QT $401 \mathrm{~ms}$ ) (data not shown). She had a normal echocardiography, and there was no history of AF in her family.

Proband 2 (male, age 45) had experienced distinct palpitations since the age of 20 years and AF was documented by ECG at the age of 33 years. At inclusion, he experienced weekly palpitations and chest discomfort lasting from one hour to half a day. The frequency of AF was higher during periods of physiological stress. The patient had a sinus rhythm ECG with IRBBB and a borderline long $\mathrm{QT}_{\mathrm{c}}$ interval where borderline prolonged in males is defined as $\mathrm{QT}_{\mathrm{c}} 440-460 \mathrm{~ms}$ [16] (Figure 1C: P-wave $80 \mathrm{~ms}$, PR $116 \mathrm{~ms}$, QRS $114 \mathrm{~ms}, \mathrm{QT}_{\mathrm{c}}$ $457 \mathrm{~ms})$. There was no sign of hypertrophy or ischemia in the ECG and echocardiography was normal. He never experienced syncope or near-syncope. For clinical purpose, the patient had an implantable loop recorder inserted for one year. The device revealed that the patient had AF $6 \%$ of the time and no ventricular arrhythmias were detected during the observation period. The patient's mother (I-3, Figure 1D) was diagnosed with paroxysmal AF and had palpitations since the age of 28 years. She also had a borderline long $\mathrm{QT}_{\mathrm{c}}$ interval of $459 \mathrm{~ms}$ and carries the mutation G60D in KCNE1. The proband's uncle (I-2) has a history of palpitations and chest discomfort, but was unavailable for genetic screening.

Both index patients were free of mutations in genes previously associated with AF (KCNQ1, KCNH2, KCNN3, KCNA5, KCNE2/3/5, KCNJ2,5, SCN5A, SCN1$3 B, A N P$, and $L M N A$ ).

KCNE1-G25V and KCNE1-G60D cause gain-of-function of $I_{K s}$ The slow delayed rectifier potassium current $I_{K s}$ is important for terminating the cardiac action potential and is composed of $K_{V} 7.1$ and KCNE1 $[17,18]$. To 


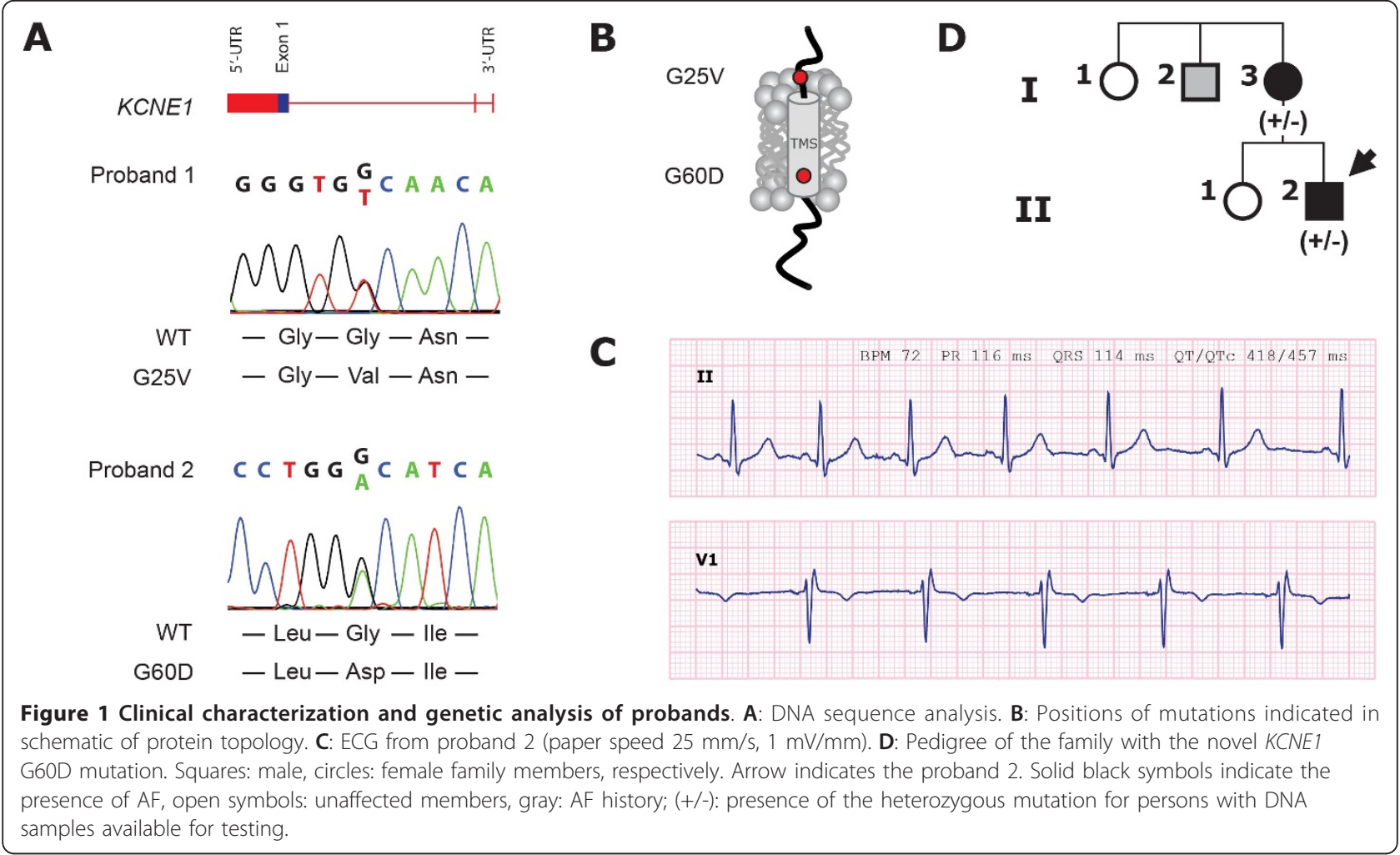

examine whether a mutation in the accessory $\beta$-subunit KCNE1 could explain the lone AF phenotype observed in the proband, we expressed wild-type (WT) or mutant KCNE1 together with the pore forming $\mathrm{K}_{\mathrm{V}} 7.1 \alpha$-subunit in X.laevis oocytes and recorded currents using the twoelectrode voltage-clamp technique.

Expression of $\mathrm{K}_{\mathrm{V}} 7.1$ and $\mathrm{KCNE} 1$ gave rise to a slowly activating and deactivating potassium current, that does not inactive (Figure 2A). Co-expression of the mutants KCNE1-G25V or KCNE1-G60D and $\mathrm{K}_{\mathrm{V}} 7.1$ (Figure 2B, Additional file 1: Figure S2) resulted in larger current amplitudes compared to the WT complex.

When summarizing the current measurements from oocytes expressing either KCNE1-WT, KCNE-G25V or KCNE-G60D together with $\mathrm{K}_{\mathrm{V}} 7.1$, subjected to $5 \mathrm{~s}$ depolarizing potentials from a holding potential of -80 $\mathrm{mV}$ we found that both mutations caused an increase in steady-state current amplitude at all activating potentials (Figure 2C, Additional file 1: Figure S2). Voltage-dependence of channel activation determined by tail-current analysis did not reveal any difference between KCNE1WT and mutants (Figure 2D, Additional file 1: Figure S2).

The KCNE1 mutations also altered biophysical properties as visible from the current traces in Figure 2. Activation of $\mathrm{K}_{\mathrm{V}} 7.1 / \mathrm{KCNE} 1$ channels results in sigmoidal activation current traces [19]. To compare the activation kinetics we determined the time needed to reach the half-maximal current level $\left(t_{1 / 2}\right)$ when currents were elicited at physiologically relevant potentials 0 and $+20 \mathrm{mV}$. The activation was significantly faster for the mutated channels as compared with wild-type channels (Figure 2E, Additional file 1: Figure S2).

Deactivation kinetics were investigated by recording tail-currents at potentials ranging from -140 to -40 in $20 \mathrm{mV}$ increments after an activating step to $+40 \mathrm{mV}$ (Figure 3A, B). The deactivating current traces were best fitted to a single exponential function. The summarized data in Figure 3C shows that expression of KCNE1G60D resulted in a significantly faster deactivation when investigated at -100 and $-120 \mathrm{mV}$. For KCNE1-G25V, deactivation kinetics were similar to WT (data not shown).

Faster activation kinetics suggests a gain-of-function in $I_{K s}$ whereas the opposite is true for the observed changes in deactivation kinetics. In the following, we focused on the functional characterization of G60D due to the more complex electrophysiological phenotype and due to the history of AF in this family. To address whether the changed channel kinetics of the mutant would result in altered heart rate-dependent modulation of $\mathrm{I}_{\mathrm{Ks}}$, protocols mimicking heart rates of 60,120 , and $180 \mathrm{bpm}$ were used. When X.laevis oocytes were subjected to voltage protocols at $60 \mathrm{bpm}$ no accumulation 


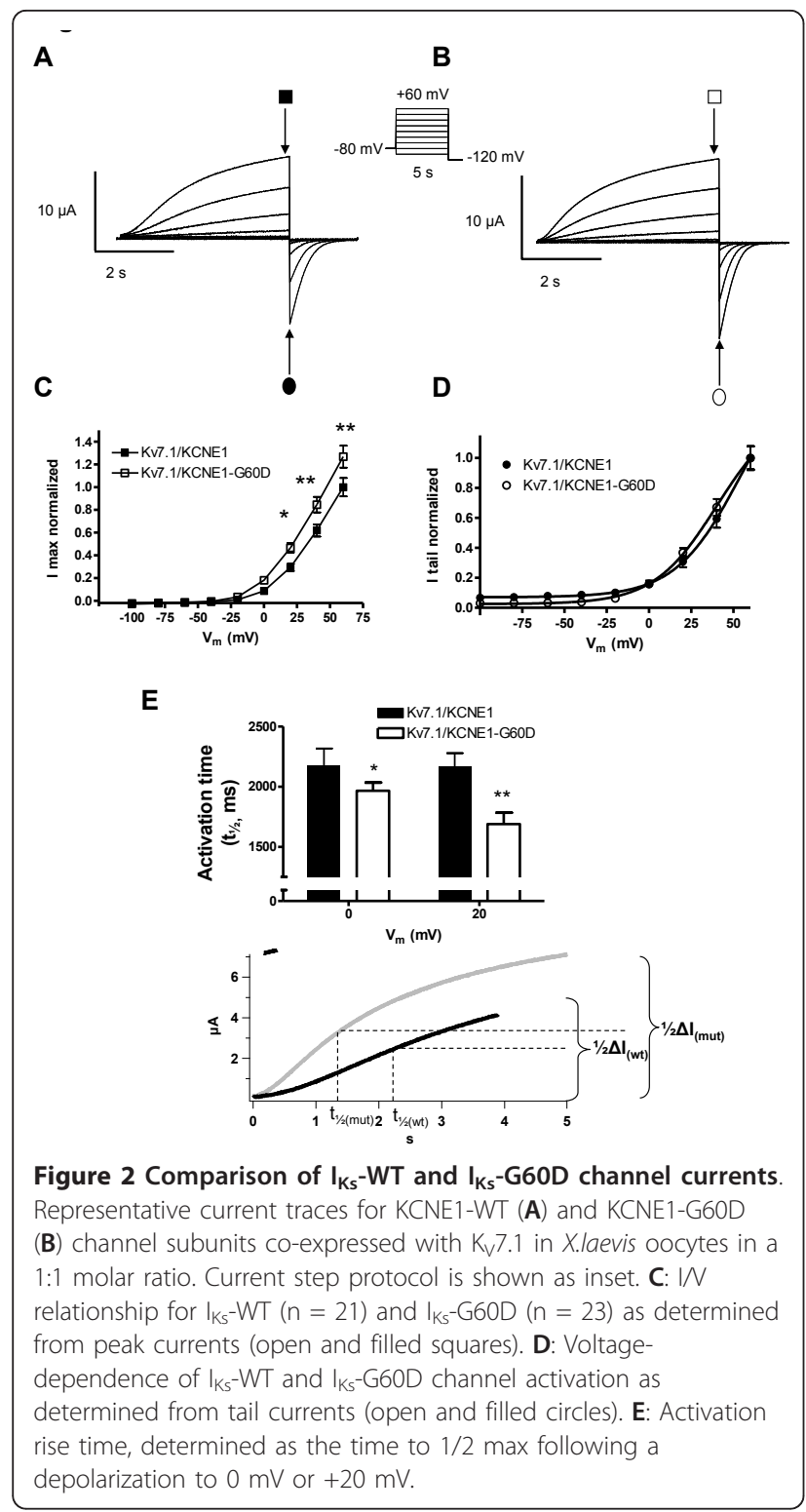

of $\mathrm{I}_{\mathrm{Ks}}$ current was observed. In comparison, "stimulation" at 120 and $180 \mathrm{bpm}$ resulted in an accumulation of $\mathrm{I}_{\mathrm{Ks}}$ current which reached significance for $180 \mathrm{bpm}$ (see Figure 4). The amount of charge conducted by $\mathrm{K}_{\mathrm{V}} 7.1 / \mathrm{KCNE} 1-\mathrm{WT}(\mathrm{n}=10)$ or $\mathrm{K}_{\mathrm{V}} 7.1 / \mathrm{KCNE} 1-\mathrm{G} 6 \mathrm{D}$ ( $\mathrm{n}$ $=15$ ) channels in the first $130 \mathrm{~ms}$ after the capacitive spike of the pulse at the 7th second was calculated and normalized to the charge carried at $60 \mathrm{bpm}$ (Figure 4). The frequency-dependent buildup of conducted charge was significantly larger in the mutated $\mathrm{I}_{\mathrm{Ks}}$ channel complex as compared to WT when measured at $180 \mathrm{bpm}$.

To mimic the heterozygote state of the affected subjects we co-expressed $\mathrm{K}_{\mathrm{V}} 7.1$ with KCNE1-WT and KCNE1-G60D. This resulted in an intermediate phenotype both with respect to current amplitudes and

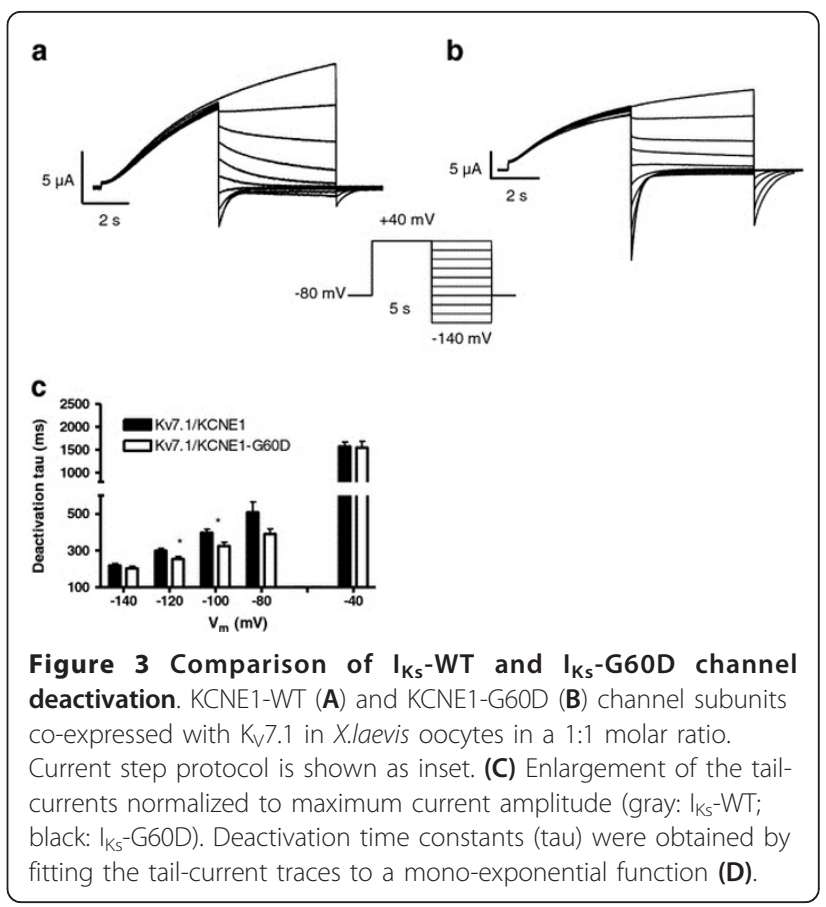

channel kinetics. Activation kinetics were not significantly different from $\mathrm{K}_{\mathrm{V}} 7.1 / \mathrm{KCNE} 1$ channels, but deactivation kinetics were significantly faster for the heterozygote state as compared to wild-type channels when measured at -120 to $-80 \mathrm{mV}$ (Additional file 1 : Figure S3).

To verify the findings from the two-electrode voltageclamp experiments, we performed whole cell patchclamp experiments in $\mathrm{CHO}-\mathrm{K} 1$ cells (Figure 5) at $36 \pm$ $1^{\circ} \mathrm{C}$. Under these conditions the expression of $\mathrm{K}_{\mathrm{V}} 7.1$ and KCNE1 resulted in a non-inactivating $\mathrm{K}_{\mathrm{V}} 7.1 / \mathrm{KCNE} 1$ current with marked faster activation and deactivation

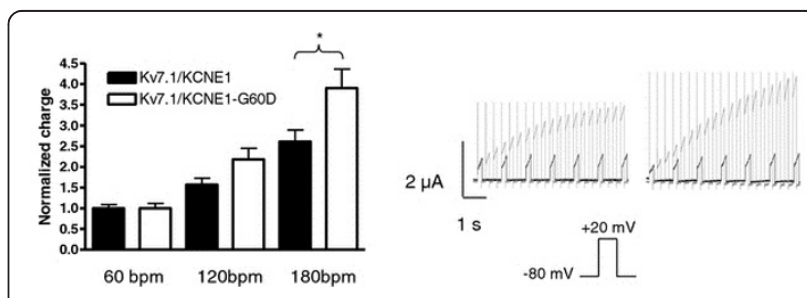

Figure 4 Frequency dependence of conduction. Three different voltage protocols were applied (60 bpm (+20 mV for $200 \mathrm{~ms}$, followed by $800 \mathrm{~ms}$ at $-80 \mathrm{mV}$ ); $120 \mathrm{bpm}(+20 \mathrm{mV}$ for $180 \mathrm{~ms}$, followed by $320 \mathrm{~ms}$ at $-80 \mathrm{mV}$ ); $180 \mathrm{bpm}$ (+20 mV for $150 \mathrm{~ms}$, followed by $180 \mathrm{~ms}$ at $-80 \mathrm{mV}$ )). The bar graph summarizes the amount of charge conducted by KV 7.1-WT/KCNE1 (black; $\mathrm{n}=10$ ) or $K_{V} 7.1 / K C N E 1-G 60 D$ (white; $n=15$ ) channels in the first $130 \mathrm{~ms}$ after the capacitive spike $(10 \mathrm{~ms}$ ) of the pulse at the 7 th second when normalized to the charge carried at $60 \mathrm{bpm}$. Representative current traces recorded (left: $K_{v} 7.1 / K C N E 1$; right: $\left.K_{v} 7.1 / K C N E 1-G 60 D\right)$ using the $60 \mathrm{bpm}$ (black) and $120 \mathrm{bpm}$ (gray) protocols are shown on the right. 


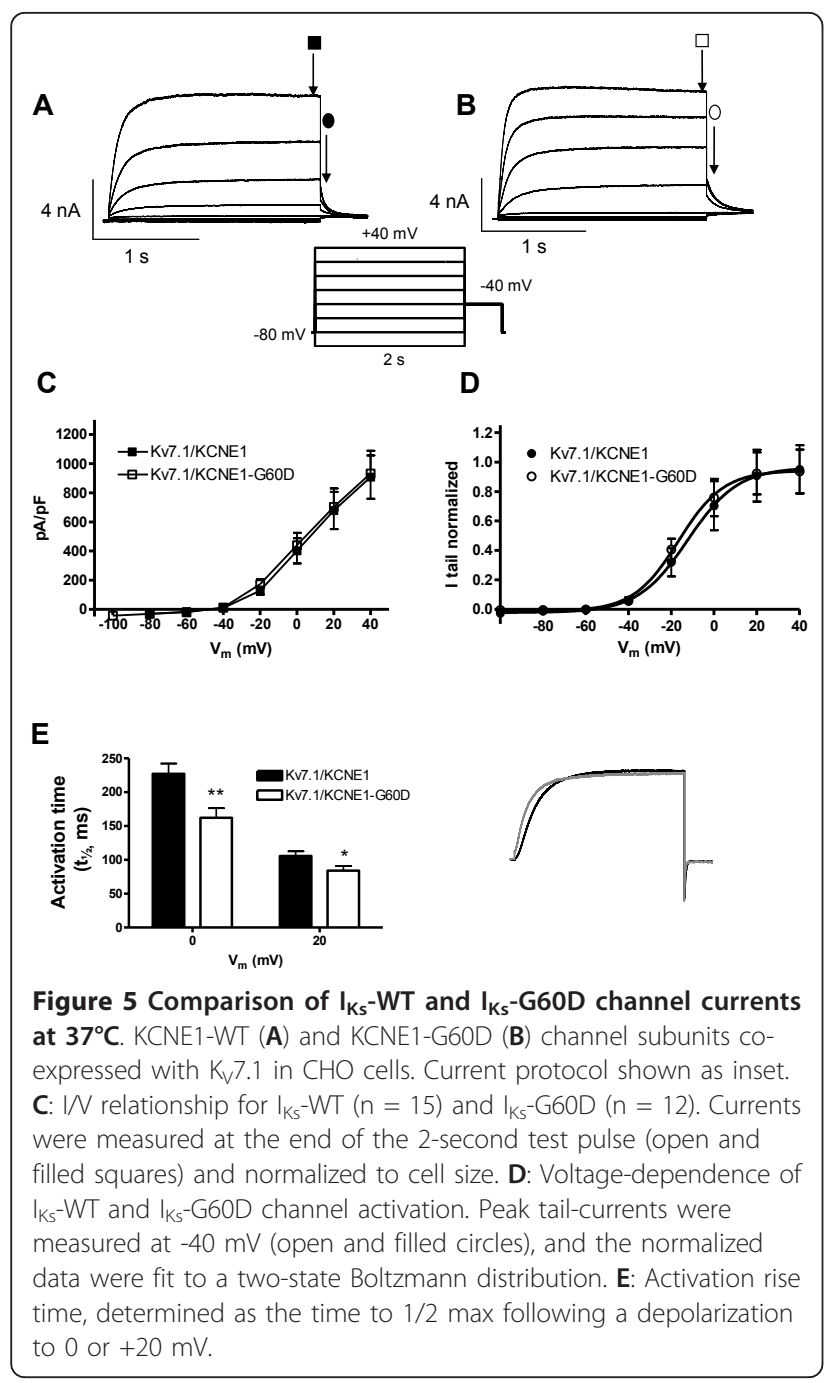

kinetics as compared to when the channel subunits were expressed in X.laevis oocytes. In the mammalian expression system we observed no significant difference between the wild-type and mutated $\mathrm{I}_{\mathrm{Ks}}$ channel complex with respect to steady-state current amplitude or voltage-dependence of channel activation. However, the activation time was still significantly faster when the mutated $\beta$-subunit was expressed together with $\mathrm{K}_{\mathrm{V}} 7.1$. No significant difference in deactivation kinetics was observed between WT and mutant (Figure 6).

\section{Discussion}

Although AF is the most common cardiac arrhythmia, the fundamental molecular pathways in many cases remain undefined. To our knowledge, the present study is the first to report that mutations in the $\beta$-subunit KCNE1 are associated with AF.

Both mutations (G25V, G60D) were absent in 216 matched controls, in publicly available databases and in
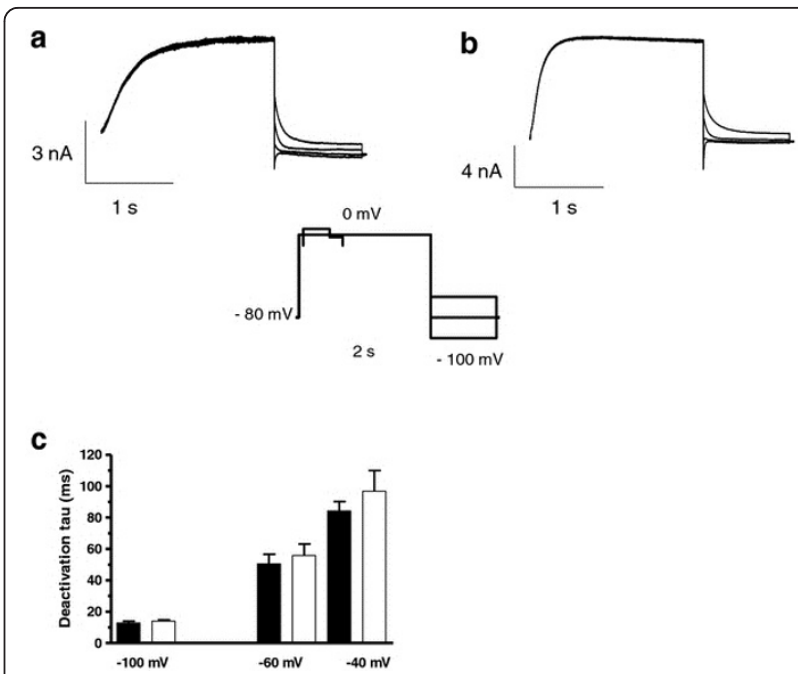

Figure 6 Comparison of $\mathrm{I}_{\mathrm{Ks}}-\mathrm{WT}$ and $\mathrm{I}_{\mathrm{Ks}}-\mathrm{G} 60 \mathrm{D}$ channel deactivation kinetics. KCNE1-WT (A) and KCNE1-G60D (B) channel subunits co-expressed with $\mathrm{K}_{\mathrm{V}} 7.1$ in $\mathrm{CHO}$ cells. Currents were elicited by clamping the cells for $2 \mathrm{~s}$ at $0 \mathrm{mV}$, followed by a $1 \mathrm{~s}$ step to test potentials ranging from -100 to $-20 \mathrm{mV}$ in $20 \mathrm{mV}$ increments, at $36 \pm 1^{\circ} \mathrm{C}$. (C) Enlargement of the tail-currents normalized to maximum current amplitude (gray: $\left.\right|_{\mathrm{Ks}}-\mathrm{WT}$; black: $\mathrm{I}_{\mathrm{Ks}}-\mathrm{G} 60 \mathrm{D}$ ).

Deactivation time constants (tau) were obtained by fitting the tailcurrent traces to a mono-exponential function (D).

2276 exoms from the Popgen population NHLBI Exome Sequencing Project http://evs.gs.washington.edu/EVS/ supporting that these variants are not rare polymorphisms but disease causing mutations [20]. The patient carrying mutation $\mathrm{G} 25 \mathrm{~V}$ had no family history of arrhythmia. The patient carrying mutation G60D had an interesting phenotype of $\mathrm{AF}$ and borderline $\mathrm{QT}_{\mathrm{c}}$ interval that co-segregated from the mother to the son.

We used the Xenopus laevis oocyte system as it allows for better control of subunit ratios compared to transfection of mammalian cell lines such as CHO-K1. In $X$. laevis oocytes, KCNE1-G25V and KCNE1-G60D showed a gain-of-function for $\mathrm{I}_{\mathrm{Ks}}$ both with respect to steadystate current levels, kinetics, and heart rate-dependent modulation of $\mathrm{I}_{\mathrm{Ks}}$ (for G60D). When investigating the mutation in an experimental condition mimicking the heterozygous state of the patient, changes were not significant and hence the functional studies did not fully explain the phenotype, which could indicate that either the mutation is not causing the phenotype, or that the interaction is more complex. Yet, we showed gain-offunction effects for both identified mutations strengthening the notion that mutations in KCNE1 are associated with AF.

Furthermore, it has been shown that monoallelic expression is much more widespread than previously thought affecting $20 \%$ of human genes [21]. In a study addressing 190 genes on chromosome 21, KCNE1 was 
found to have $10 \mathrm{CG}$ methylation sites rendering KCNE1 a profoundly epigenetically regulated gene [22] thereby making also the homozygote experimental condition highly relevant.

Though differences were less pronounced in mammalian cells, we still observed a gain-of-function of activation kinetics for G60D. Mutations leading to even mild gain-of-function of the $\mathrm{I}_{\mathrm{Ks}}$ current have been described earlier in the context of lone AF, yet all mutations identified so far reside in the $\alpha$-subunit of the channel complex $[18,23]$. Two studies have investigated a possible association between AF and the SNP G38S in the $\beta$-subunit KCNE1 in Chinese AF cohorts. Lai et al. reported a significant association of $38 \mathrm{G}$, which was not found in the later study $[24,25]$. Of note, others observed decreased $\mathrm{I}_{\mathrm{Ks}}$ amplitudes and reduced surface expression with $38 \mathrm{G}$ pointing to a loss-of-function of this variant [26].

The mutation G25V is located in the extracellular Nterminus of the channel protein. A recent study as implicated the extracellular juxtamembranous region of KCNE1 in gating [27], however, little is known about the role of the proximal KCNE1 N-terminus. The mutation G60D resides in the transmembrane segment (TMS) of KCNE1 in close proximity of residues 57-59 ("the gating triplet") that are critical for the modulation of $K_{V} 7.1$ channels [28]. Several glycine residues located in the TMS including G60 seem instrumental for forming a curvature that locates threonine 58 [29], which is critical for slow activation kinetics conferred by KCNE1 [30]. Hence, the mutation G60D in this close proximity could be speculated to compromise the function of this critical amino acid and thereby speed up activation [30-33].

Gain-of-function mutations of the $\mathrm{I}_{\mathrm{Ks}}$ channel are expected to increase the repolarising potassium currents which could abbreviate the cardiac action potential duration as well as the effective refractory period in cardiomyocytes. The mutation could thereby create a profibrillatory substrate within the atrium $[10,11] . \mathrm{I}_{\mathrm{Ks}}$ also plays a major role in ventricular repolarization. Gain-offunction of $\mathrm{I}_{\mathrm{Ks}}$ would be expected to result in both action potential and $\mathrm{QT}_{\mathrm{c}}$ shortening which we did not observe in the proband. It has been suggested that common variations in other genes may protect the patient from a shortening of $\mathrm{QT}_{\mathrm{c}}$ [31]. All mutation carriers in this study had not experienced ventricular arrhythmias, yet display a borderline long $\mathrm{QT}_{\mathrm{c}}$ interval. Pai and Rawles suggested a link between $\mathrm{AF}$ and prolongation of the mean QT interval [32]. The first study associating gainof-function mutations in $\mathrm{K}_{\mathrm{V}} 7.1$ with $\mathrm{AF}$ analyzed a large four-generation family. Nine of 16 family members diagnosed with autosomal dominant hereditary AF showed prolonged $\mathrm{QT}_{\mathrm{c}}$ ranging from 450 to $530 \mathrm{~ms}$ [33]. Vice versa, Ackermann and colleagues reported presence of early-onset AF in a cohort of congenital LQT patients underlining the evidence for a link between these two syndromes [34]. Very recently, also a Brugada syndrome associated gain-of-function mutation in $\mathrm{K}_{\mathrm{V}} 4.3$, the $\alpha$ subunit underlying the transient outward current $\mathrm{I}_{\mathrm{to}}$, has been linked with $\mathrm{QT}_{\mathrm{c}}$ prolongation in the affected family member [35].

Despite the fact that proband 2 and his mother, both carrying the mutation $\mathrm{G} 60 \mathrm{D}$, had borderline long $\mathrm{QT}_{\mathrm{c}}$ intervals, there was no further suspicion of LQTS in the two patients. Clinical evaluation revealed no family history of syncope or near-syncope and the proband had a one year implantable loop recorder without any ventricular arrhythmias detected. Of note, both our index probands had an ECG pattern of IRBBB, which has recently been shown to be associated with early-onset lone AF [15] supporting the AF phenotype in the patients.

The exact mechanisms linking AF and QT prolongation and the different effect of $\mathrm{I}_{\mathrm{Ks}}$ mutations remain to be elucidated. One possible explanation may be different composition of the $\mathrm{I}_{\mathrm{Ks}}$ channel complex in atria and ventricles as suggested earlier to explain a mixed phenotype of AF and QT prolongation in a patient with a KCNQ1 mutation [18]. Also, chamber-specific interaction partners yet to be identified may modulate the effects of mutations in atria and ventricles. Furthermore, differences in triggers such as the well-documented beta-adrenergic stimulation [36] or the more recently described modulation by natriuretic peptide precursor $\mathrm{A}$ may be involved [23,37].

\section{Limitations}

We limited our analysis to the KCNE1 encoding regions, and the possibility of mutations occurring in regions of the gene other than coding regions cannot be excluded. Though we performed genetic testing of all genes associated with AF earlier, we cannot exclude mutations in yet unknown genes. Furthermore, genetic testing of family members was limited as some were unavailable. Also, the number of probands was small, however, it should be noted that the cohort of young lone AF patients was well-defined. The functional analyses used conventional heterologous expression systems in which the environments differ from that in the native cardiomyocytes.

\section{Conclusions}

In this study of lone AF patients, we found two suspected disease-causing mutations in KCNE1. Functional analysis of G25V and G60D showed a gain-of-function of the $\mathrm{I}_{\mathrm{Ks}}$ current. This study supports the hypothesis that gain-of-function in potassium current enhances AF susceptibility. 


\section{Additional material}

Additional file 1: The additional file (PDF) contains a detailed description of the methods, three additional figures (Additional file 1: Figure S1: Conservation of KCNE1 G25 and G60 within different species; Additional file 1: Figure S2: Comparison of $\mathrm{I}_{\mathrm{Ks}}-\mathrm{WT}$ and $\mathrm{I}_{\mathrm{Ks}}$ G25V channel currents in Xenopus laevis oocytes; Additional file 1: Figure S3: Comparison of $I_{\mathrm{Ks}}-\mathrm{WT}$ and $\mathrm{I}_{\mathrm{Ks}}-\mathrm{G} 60 \mathrm{D}$ and $\mathrm{I}_{\mathrm{Ks}}-\mathrm{WT} / \mathrm{G} 60 \mathrm{D}$ channel currents.), and additional references.

\begin{abstract}
Acknowledgements
We are grateful to the patients and the cardiology departments at Frederiksberg, Hvidovre, Gentofte, Glostrup, Amager, Herlev and Bispebjerg hospitals for help with supply of patient data. We thank Pia Hagman and Amer Mujezinovic for technical assistance.

Funding sources

The Danish National Research Foundation, The John and Birthe Meyer Foundation, The Research Foundation of the Heart Centre Rigshospitalet, The Arvid Nilsson Foundation, and Director lb Henriksens Foundation.
\end{abstract}

\section{Author details}

${ }^{1}$ The Danish National Research Foundation Centre for Cardiac Arrhythmia, Copenhagen, Denmark. ²Laboratory for Molecular Cardiology, The Heart Centre, Rigshospitalet, University of Copenhagen, Copenhagen, Denmark. ${ }^{3}$ The Ion Channel Group, Department of Biomedical Sciences, Faculty of Health Sciences, University of Copenhagen, Blegdamsvej 3, 12.5.14, Copenhagen, N 2200, Denmark. ${ }^{4}$ Department of Cardiology, Aarhus University Hospital, Skejby, Denmark. ${ }^{5}$ Department of Surgery and Medicine, Faculty of Health Sciences, University of Copenhagen, Copenhagen, Denmark.

\section{Authors' contributions}

MSO performed mutation screening, participated in the study design and wrote the manuscript. BHB participated in the study design, performed heterologous expression studies for G60D and the statistical analysis, and drafted the manuscript. JBN acquired clinical data and drafted the manuscript. ABS and JPD performed heterologous expression studies for G25V. JJ participated in the mutation screening. HKJ, SH and JHS acquired clinical data and helped to draft the manuscript. NS performed molecular biology and sequence alignment, conceived the study, participated in its design and coordination and wrote the manuscript. All authors read and approved the final manuscript.

\section{Competing interests}

The authors declare that they have no competing interests.

Received: 3 October 2011 Accepted: 3 April 2012 Published: 3 April 2012

\section{References}

1. Benjamin EJ, Wolf PA, D'Agostino RB, et al: Impact of atrial fibrillation on the risk of death: the Framingham heart study. Circulation 1998, 98(10):946-952.

2. Fuster V, Rydén LE, Cannom DS, et al: ACC/AHA/ESC 2006 guidelines for the management of patients with atrial fibrillation-excutive summary. Rev Port Cardiol 2007, 26(4):383-446

3. Stewart S, Hart CL, Hole DJ, McMurray JJ: Population prevalence, incidence, and predictors of atrial fibrillation in the Renfrew/Paisley study. Heart 2001, 86(5):516-521.

4. Go AS, Hylek EM, Phillips KA, et al: Prevalence of diagnosed atrial fibrillation in adults: national implications for rhythm management and stroke prevention: the anticoagulation and risk factors in atrial fibrillation (ATRIA) study. JAMA 2001, 285(18):2370-2375.

5. Psaty BM, Manolio TA, Kuller LH, et al: Incidence of and risk factors for atrial fibrillation in older adults. Circulation 1997, 96(7):2455-2461.

6. Mahida S, Lubitz SA, Rienstra M, Milan DJ, Ellinor PT: Monogenic Atrial Fibrillation as Pathophysiological Paradigms. Cardiovasc Res 2010, Available at: http://cardiovascres.oxfordjournals.org/content/early/2010/11/ 30/cvr.cvq381.abstract. Åbnet December 21, 2010.
7. Gudbjartsson DF, Arnar DO, Helgadottir A, et al: Variants conferring risk of atrial fibrillation on chromosome 4q25. Nature 2007, 448(7151):353-357.

8. Olesen MS, Holst AG, Jabbari J, et al: Genetic Loci on Chromosomes 4q25, 7p31, and 12p12 Are Associated With Onset of Lone Atrial Fibrillation Before the Age of 40 Years. The Canadian Journal of Cardiology 2012 [http://www.ncbi.nlm.nih.gov/pubmed/22336519].

9. Olesen MS, Jespersen T, Nielsen JB, et al: Mutations in sodium channel $\beta$ subunit SCN3B are associated with early-onset lone atrial fibrillation. Cardiovasc Res 2011, 89(4):786-793.

10. Nattel S: New ideas about atrial fibrillation 50 years on. Nature 2002, 415(6868):219-226.

11. Moe GK: Evidence for reentry as a mechanism of cardiac arrhythmias. Rev Physiol Biochem Pharmacol 1975, 72:55-81.

12. Yang Y, Li J, Lin X, et al: Novel KCNA5 loss-of-function mutations responsible for atrial fibrillation. J Hum Genet 2009, 54(5):277-283.

13. Lundby A, Tseng G-N, Schmitt N: Structural basis for K(V)7.1-KCNE(x) interactions in the I(Ks) channel complex. Heart Rhythm 2010, 7(5):708-713.

14. Olesen MS, Jabbari J, Holst AG, et al: Screening of KCNN3 in patients with early-onset lone atrial fibrillation. Europace 2011, Available at: http://www. ncbi.nlm.nih.gov/pubmed/21398315 Åbnet April 18, 2011.

15. Nielsen JB, Olesen MS, Tangø M, et al: Incomplete right bundle branch block: a novel electrocardiographic marker for lone atrial fibrillation. Europace 2011, 13(2):182-187.

16. Johnson JN, Ackerman MJ: QTc: how long is too long? Br J Sports Med 2009, 43(9):657-662.

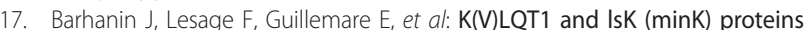
associate to form the I(Ks) cardiac potassium current. Nature 1996, 384(6604):78-80.

18. Lundby A, Ravn LS, Svendsen JH, Olesen S-P, Schmitt N: KCNQ1 mutation Q147R is associated with atrial fibrillation and prolonged QT interval. Heart Rhythm 2007, 4(12):1532-1541.

19. Sanguinetti MC, Curran ME, Zou A, et al: Coassembly of K(V)LQT1 and minK (IsK) proteins to form cardiac I(Ks) potassium channel. Nature 1996, 384(6604):80-83.

20. Anon: Exome Variant Server, NHLBI Exome Sequencing Project (ESP), Seattle, WA:[http://evs.gs.washington.edu/EVS/], [10 juli, 2011 accessed].

21. Serre D, Gurd S, Ge B, et al: Differential allelic expression in the human genome: a robust approach to identify genetic and epigenetic Cisacting mechanisms regulating gene expression. PLoS Genet 2008, 4(2): e1000006.

22. Zhang Y, Rohde C, Tierling S, et al: DNA methylation analysis of chromosome 21 gene promoters at single base pair and single allele resolution. PLOS Genet 2009, 5(3):e1000438.

23. Abraham RL, Yang T, Blair M, Roden DM, Darbar D: Augmented potassium current is a shared phenotype for two genetic defects associated with familial atrial fibrillation. J Mol Cell Cardiol 2010, 48(1):181-190.

24. Zeng Z, Tan C, Teng S, et al: The single nucleotide polymorphisms of I(Ks) potassium channel genes and their association with atrial fibrillation in a Chinese population. Cardiology 2007, 108(2):97-103.

25. Lai L-P, Su M-J, Yeh H-M, et al: Association of the human minK gene $38 \mathrm{G}$ allele with atrial fibrillation: evidence of possible genetic control on the pathogenesis of atrial fibrillation. Am Heart J 2002, 144(3):485-490.

26. Ehrlich JR, Zicha S, Coutu P, Hébert TE, Nattel S: Atrial fibrillationassociated minK38G/S polymorphism modulates delayed rectifier current and membrane localization. Cardiovasc Res 2005, 67(3):520-528.

27. Wang $Y H$, Jiang $M, X u X L$, et al: Gating-related molecular motions in the extracellular domain of the IKs channel: implications for IKs channelopathy. J Membr Biol 2011, 239(3):137-156.

28. Melman YF, Domènech A, de la Luna S, McDonald TV: Structural determinants of KvLQT1 control by the KCNE family of proteins. J Biol Chem 2001, 276(9):6439-6444.

29. Kang C, Tian C, Sönnichsen FD, et al: Structure of KCNE1 and implications for how it modulates the KCNQ1 potassium channel. Biochemistry 2008, 47(31):7999-8006.

30. Melman YF, Krumerman A, McDonald TV: A single transmembrane site in the KCNE-encoded proteins controls the specificity of KvLQT1 channel gating. J Biol Chem 2002, 277(28):25187-25194.

31. Newton-Cheh $C$, Eijgelsheim M, Rice KM, et al: Common variants at ten loci influence QT interval duration in the QTGEN Study. Nat Genet 2009, 41(4):399-406. 
32. Pai GR, Rawles JM: The QT interval in atrial fibrillation. Br Heart J 1989, 61(6):510-513.

33. Chen Y-H, Xu S-J, Bendahhou S, et al: KCNQ1 gain-of-function mutation in familial atrial fibrillation. Science 2003, 299(5604):251-254.

34. Johnson JN, Tester DJ, Perry J, et al: Prevalence of early-onset atrial fibrillation in congenital long QT syndrome. Heart Rhythm 2008, 5(5):704-709.

35. Giudicessi JR, Ye D, Tester DJ, et al: Transient Outward Current (Ito) Gainof-Function Mutations in the KCND3-Encoded Kv4.3 Potassium Channel and Brugada Syndrome. Heart Rhythm, Available at: http://www. sciencedirect.com/science/article/B7GW9-527GJC8-2/2/ 89c6a7e6ed6b2ab449934bf44708dfc8 Åbnet April 2, 2011.

36. Terrenoire C, Clancy CE, Cormier JW, Sampson KJ, Kass RS: Autonomic control of cardiac action potentials: role of potassium channel kinetics in response to sympathetic stimulation. Circ Res 2005, 96(5):e25-e34.

37. Shimizu K, Shintani Y, Ding W-G, Matsuura H, Bamba T: Potentiation of slow component of delayed rectifier $\mathrm{K}(+)$ current by CGMP via two distinct mechanisms: inhibition of phosphodiesterase 3 and activation of protein kinase G. Br J Pharmacol 2002, 137(1):127-137.

\section{Pre-publication history}

The pre-publication history for this paper can be accessed here: http://www.biomedcentral.com/1471-2350/13/24/prepub

doi:10.1186/1471-2350-13-24

Cite this article as: Olesen et al:: Mutations in the potassium channel subunit KCNE1 are associated with early-onset familial atrial fibrillation. BMC Medical Genetics 2012 13:24.

\section{Submit your next manuscript to BioMed Central} and take full advantage of:

- Convenient online submission

- Thorough peer review

- No space constraints or color figure charges

- Immediate publication on acceptance

- Inclusion in PubMed, CAS, Scopus and Google Scholar

- Research which is freely available for redistribution

Submit your manuscript at www.biomedcentral.com/submit 